\title{
A Atrial fibrillation and atrial flutter in athletes
}

\section{OPEN ACCESS}

${ }^{1}$ Arrhythmia Unit, Department of Cardiology and Cardiovascular Surgery,

University of Navarra, Navarra, Pamplona, Spain

${ }^{2}$ Thorax Institute, Hospital Clínic, University of Barcelona, Institut d'Investigació Biomèdica August Pi i Sunyer (IDIBAPS), Barcelona, Catalonia, Spain

\section{Correspondence to}

Dr Lluís Mont, Thorax Institute (ICT) —Cardiology Department, Hospital Clinic, University of Barcelona, Villarroel 170,

Barcelona 08036, Catalonia, Spain;

Imont@clinic.ub.es

Accepted 30 May 2012

\author{
Naiara Calvo, ${ }^{1}$ Josep Brugada, ${ }^{2}$ Marta Sitges, ${ }^{2}$ Lluís Mont ${ }^{2}$
}

\section{ABSTRACT}

Atrial fibrillation (AF) is the most common arrhythmia in clinical practice, with an estimated prevalence of $0.4 \%$ to $1 \%$ in the general population, increasing with age to $8 \%$ in those above 80 years. The recognised risk factors for developing AF include age, structural heart disease, hypertension, diabetes mellitus or hyperthyroidism. However, the mechanisms underlying the initiation of $A F$ in patients below 60 years of age, in whom no cardiovascular disease or any other known causal factor is present, remain to be clarified. This condition, termed as lone $\mathrm{AF}$, may be responsible for as many as $30 \%$ of patients with paroxysmal AF seeking medical attention. Recent studies suggest that long-term endurance exercise may increase the incidence of $A F$ and atrial flutter (AFI) in this population. This review article is intended to analyse the prevalence of $\mathrm{AF}$ and $\mathrm{AFI}$, the pathophysiological mechanisms responsible for the association between endurance sport practice and AF or AFI and the recommended therapeutic options in endurance athletes.

\section{INTRODUCTION}

Atrial fibrillation (AF) is the most common clinically significant cardiac arrhythmia in clinical practice. The prevalence of AF among men below 40 years of age in the general population is $0.5 \%,{ }^{1}$ and increases to $8 \%$ in those above 80 years. ${ }^{2}$ Several cardiac and non-cardiac conditions, including age, structural heart disease, hypertension, diabetes mellitus and hyperthyroidism, ${ }^{3}$ have been described as risk factors for developing AF. However, in a subset of patients with AF younger than 60 years, routine evaluation including physical examination, laboratory tests including thyroid function, echocardiography and exercise stress testing does not reveal any cardiovascular disease or any other known causal factor. These patients are considered to suffer from 'lone' AF (LAF). ${ }^{4}$ The reported prevalence of LAF varies from $2 \%$ to $50 \%$, depending on the chosen study population with $\mathrm{AF}^{5} 6$

Although benefits of regular exercise on reduction of the risks of cardiovascular diseases have been demonstrated, ${ }^{7}{ }^{8}$ there is growing evidence that long-term endurance exercise may increase the risk of developing $\mathrm{AF}$ and atrial flutter (AFI) in middle-aged populations. ${ }^{9-17}$

This article discusses the prevalence of AF and AFl, pathophysiological mechanisms, clinical presentation and treatment strategies in endurance athletes.

\section{AF AND ENDURANCE SPORT}

Several studies have described a relationship between long-term endurance sport practice and $\mathrm{AF}$ and $\mathrm{AFl}^{9-20}$ (table 1). In 1998, Karjalainen et al ${ }^{9}$ concluded that vigorous long-term exercise is associated with AF in healthy middle-aged men They evaluated AF prevalence in veteran male orienteers and in a matched control group. LAF was diagnosed in 12 of 228 (5.3\%) orienteers and in 2 of $212(0.9 \%)$ controls (who also engaged in vigorous exercise $)(p=0.012)$, the relative risk being 5.5 (95\% CI 1.3 to 24.4 ) in orienteers. Our group analysed 1160 consecutive patients seen at the Outpatient Arrhythmia Clinic between October 1997 and March 1999. The proportion of sport activity among patients with LAF was significantly higher than among men from the general population in Catalonia $(62.7 \%$ vs $15.4 \%) .{ }^{10}$ Regular sport activity was defined as high-intensity practice for at least $3 \mathrm{~h}$ a week for 2 years. An age-matched study including the same population of athletic men with LAF and agematched controls selected from the general population of Girona, using data from the REGICOR (Registre Gironí del Cor) Study, confirmed that current and prolonged sport practice, defined as more than 1500 lifetime hours of intense endurance practice, was associated with a three times higher prevalence of LAF, and with five times higher prevalence of vagal LAF (OR 5.06, 95\% CI 1.35 to 19$).{ }^{12}$ Baldesberger et al ${ }^{13}$ published similar data in a study of 62 professional cyclists who completed the Tour de Suisse professional cycling race at least once during the years 1955-1975. These cyclists were matched for age, weight, hypertension and cardiac medication with a control group of 62 male golfers who had never performed high-endurance training. The incidence of AF and AFl was significantly higher among athletes. Heidbuchel et al ${ }^{14}$ analysed the relationship between a history of endurance sports activity and/or its continuation and the risk of developing $\mathrm{AF}$ in patients with AFl undergoing right isthmus ablation. Of the 137 patients included, 31 (23\% of the whole population) were mainly engaged in endurance activities. A history of endurance sports participation was an independent risk factor for AF development after flutter ablation (multivariate HR 1.81, 95\% CI 1.10 to 2.98) and ongoing practice of an endurance sport after AFl ablation also increased the risk of AF (multivariate HR 1.68, 95\% CI 0.92 to 3.06 ).

In a further cohort study, we evaluated the incidence of LAF in 183 individuals who ran the Barcelona Marathon in 1992 in comparison to 290 sedentary healthy individuals. The incidence of LAF was higher among marathon runners compared to sedentary men (annual incidence: 0.43/ 100 for runners, $0.11 / 100$ for sedentary men) at 10 years of follow-up. ${ }^{15}$ In the GIRAFA (Grup Integrat de Recerca en Fibril-lacio Auricular) study, ${ }^{16}$ our group recruited patients with recent onset LAF attending the emergency room at our 
Table 1 Summary of the published studies analysing the relationship between atrial fibrillation and atrial flutter and endurance sport practice

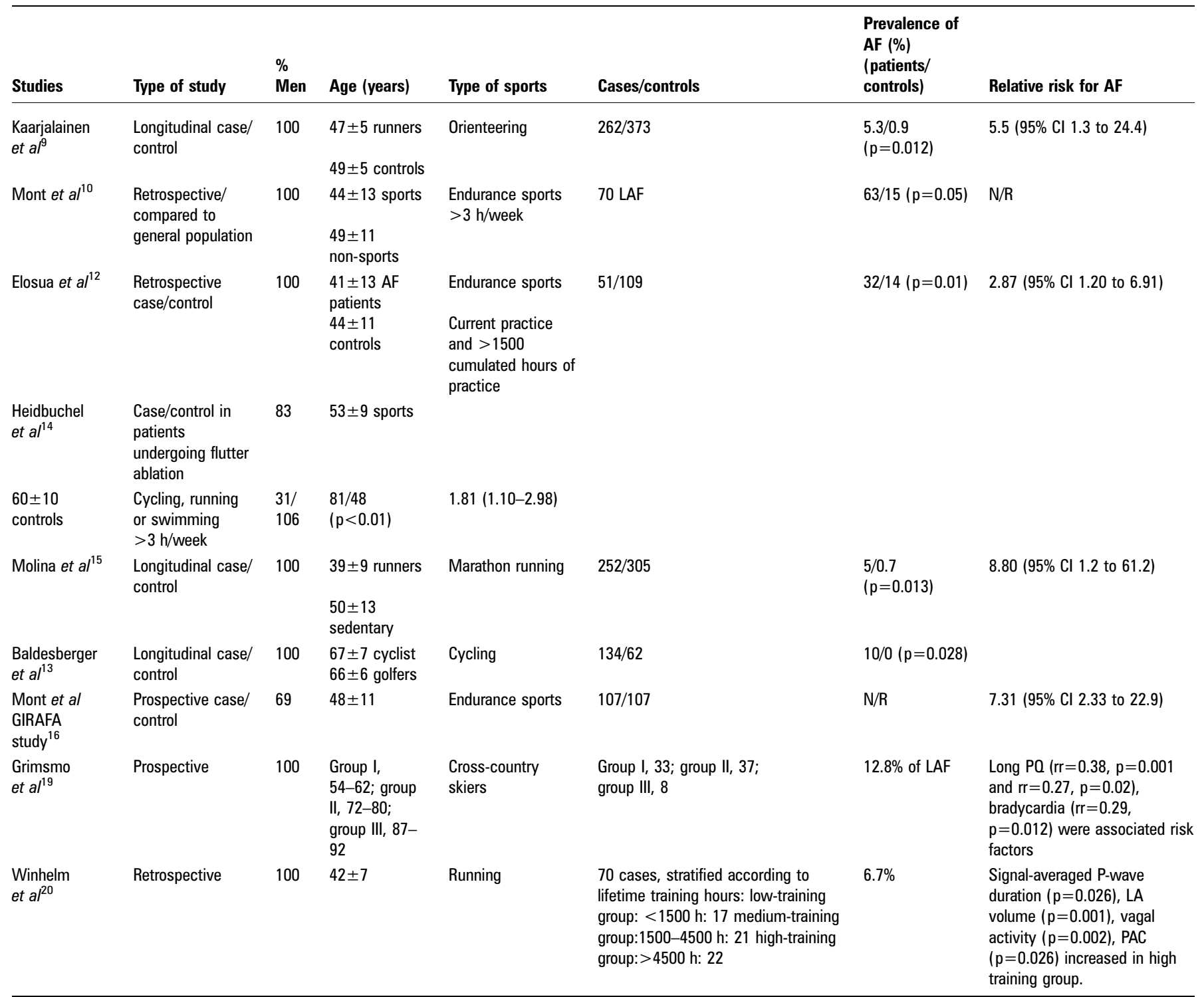

$L A$, left atrial; $P A C$, premature atrial contractions.

hospital between January 2001 and June 2005. They were matched by age and sex with healthy controls. An association of LAF and accumulated hours of physical activity was described: intense physical activity of $>564 \mathrm{~h}$ was associated with a risk for developing AF of 7.31 (95\% CI 2.33 to 22.96).

A recent meta-analysis by Abdulla and Nielsen ${ }^{17}$ demonstrated that the overall risk for AF was significantly higher in athletes than in controls (OR 5.29, 95\% CI 3.57 to 7.85). Additionally, these results were confirmed by a large prospective cohort study of apparently healthy men. ${ }^{18}$ After adjustment for multiple potentially confounding lifestyle factors and health conditions, Aizer et al showed a $20 \%$ increased risk of developing AF among individuals with higher frequency of participation in a regular programme of vigorous exercise. More recently, Grimsmo et al ${ }^{19}$ analysed the prevalence of LAF in 117 crosscountry skiers who competed in the Norwegian 'Birkebeiner' race of $58 \mathrm{~km}$. They found that the prevalence of LAF was approximately $13 \%$ and bradycardia and long $\mathrm{PQ}$ time were independent predictors for the occurrence of LAF. Winhelm et $a l^{20}$ recruited non-elite athletes participating in The Grand
Prix of Bern, one of the most popular 10-mile races in Switzerland, and found that the prevalence of AF was $6.7 \%$.

In contrast to these previous studies, Pelliccia et $a l^{21}$ analysed the frequency of AF and supraventricular tachycardias in 1777 highly trained athletes. They reported a low incidence of AF among competitive athletes $(0.2 \%)$, similar to that observed in general populations of comparable age and sex. However, in contrast to previous studies showing an association between $\mathrm{AF}$ and long-term endurance sport practice, the population analysed by Pelliccia et al comprised young athletes (mean age $24 \pm 6$ years) involved in vigorous training programmes for a mean time period of only 6 years.

On the other hand, in the Cardiovascular Health Study, ${ }^{22}$ the incidence of AF in older adults ( $>65$ years old) was lower with moderate-intensity exercise. However, this was not true with high-intensity exercise.

In summary, previous studies support an association between long-term endurance sports practice and the occurrence of arrhythmias such as AF or AFl in the middle-aged male population. 


\section{AFL AND ENDURANCE SPORT ACTIVITY}

Many of the described series report the presence of both AF and AFl in endurance athletes. Hoogsteen et al ${ }^{11}$ found that AFl was present in $10 \%$ of athletes with paroxysmal AF. Baldesberger et $a l^{13}$ evaluated arrhythmias in a long-term follow-up (30-50 years) after high endurance training in former professional cyclists, and found that AFI was more common than AF.

Heidbuchel et al ${ }^{14}$ described a higher incidence of AF after common flutter ablation in endurance athletes than in controls. According to these authors, flutter ablation could unmask the underlying atrial disease in endurance athletes, resulting in AF development during follow-up.

Based on these findings, endurance sport may contribute to the development of both arrhythmias.

\section{PATHOPHYSIOLOGY OF AF AND AFL IN ENDURANCE ATHLETES}

The pathophysiological mechanisms responsible for the increased risk of AF in individuals who practice an endurance sport remain speculative. Atrial ectopic beats, inflammatory changes, changes in electrolytes, atrial enlargement with dilatation and fibrosis and increased vagal tone and bradycardia, among others, have been proposed as mechanisms.

\section{Atrial ectopic beats}

Conflicting data exist regarding increased atrial ectopy with physical activity. It is accepted that pulmonary vein ectopy may be the trigger in episodes of paroxysmal $\mathrm{AF}^{23}$ Since atrial ectopy has been shown to be increased as a consequence of physical activity, ${ }^{20} 24$ it has been proposed that increased atrial ectopy might also explain the increased risk of AF associated with sport practice. However, these findings were not supported by Baldesberger et al, ${ }^{13}$ they did not find an increased incidence of atrial ectopy, despite increases in ventricular ectopy in former professional cyclists.

\section{Influence of the autonomic nervous system}

The role of the cardiac autonomic nervous system in the initiation and maintenance of AF has been widely investigated. ${ }^{25-28}$ In an elegant study, Coumel ${ }^{29}$ analysed the influence of autonomic innervations in patients with frequent attacks of paroxysmal AF and AFl; they found that two opposite patterns and mechanisms (vagal and sympathetic, which often interact) can be identified. According to these studies, the vagal influences predominated in normal atria, and the formation of macroreentrant circuits such as flutter might be explained by the shortening of the wavelength of the atrial impulse as a consequence of vagal stimulation. Diseased atria, however, were more dependent on adrenergic influences, which favoured the formation of microreentries and automatic and triggered activities. Vagal AF was originally described as AF that (1) predominantly affects males between 30 and 50 years of age, (2) usually occurs at night and rarely occurs between breakfast and lunch when the sympathetic tone is high, (3) rarely occurs during exercise or emotional stress, (4) is frequently triggered during relaxation after stress and (5) is often preceded by bradycardia.

Most of the available data support the association between sport practice and LAF, implicating increased vagal tone as the principal underlying mechanism. According to the GIRAFA study, ${ }^{16}$ vagal AF was the most common form of LAF: $70 \%$ of consecutive patients with LAF had vagal AF. Grimsmo et al ${ }^{19}$ found that bradycardia and long $\mathrm{PQ}$ time were predictors for the occurrence of LAF. Winhelm et $a l^{20}$ showed that athletes in the high training group ( $>4500 \mathrm{~h}$ of lifetime training) had a significantly greater parasympathetic tone (figure 1). Therefore, the increased vagal tone induced by endurance sport practice might explain the appearance of AF.

\section{Exercise and structural changes in the atrium}

It is well known that the athlete's heart, although assumed to be a physiological adaptation, has increased atrial size and ventricular mass and altered diastolic function, which may create a favourable substrate for the disease.

Frustaci et $a l^{30}$ analysed the structural changes in the atria of patients with LAF. They described the presence of inflammatory lymphonomonuclear infiltrates, compatible with myocarditis, non-inflammatory cardiomyopathic processes and patchy fibrosis. However, in the literature data regarding the cardiac histological and biochemical remodelling in endurance athletes are scarce.

\section{Fibrosis}

Our group analysed the morphological changes in an experimental study in male Wistar rats. ${ }^{31}$ A group of rats was conditioned to run vigorously for 4, 8 and 16 weeks and compared to time-matched sedentary rats that served as controls. At 16 weeks of training, exercise rats developed eccentric hypertrophy and diastolic dysfunction as well as atrial dilation with collagen deposition at the atria and the right ventricle. An increase in mRNA and protein expression of a series of fibrotic markers in the right ventricle and in both atria was found in exercise rats compared to sedentary rats.

A case-control study by Lindsay and Dunn ${ }^{32}$ analysed the presence of humoral markers of fibrosis in 45 veteran athletes compared to sedentary subjects. Athletes showed an increase in three collagen markers-plasma PICP, CITP, and TIMP-1suggesting that long-term sport practice may provoke fibrosis as part of the hypertrophic process in veteran athletes.

More recently, Breuckmann et al ${ }^{33}$ prospectively analysed the myocardial distribution of late gadolinium enhancement with delayed-enhancement cardiac magnetic resonance imaging in 102 non-professional male marathon runners and in asymptomatic age-matched control subjects. A three times higher rate of myocardial damage was found among runners compared to the sedentary control group ( $12 \%$ vs $4 \% ; p=0.077)$. Similar findings were reported by Wilson et al, ${ }^{34}$ who described a high prevalence $(50 \%)$ of myocardial fibrosis in healthy, asymptomatic, veteran, male lifelong athletes, compared to zero cases in agematched veteran controls and young athletes.

\section{Myocardial injury}

Several studies have demonstrated elevations in highly specific cardiac biomarkers (cardiac troponin $\mathrm{T}$ and $\mathrm{I}$ ) after prolonged exercise, suggesting that strenuous physical exertion may result in myocardial injury. ${ }^{35-40}$ A recent meta-analysis by Shave et $a l^{41}$ demonstrated that the incidence of post-exercise cTnT release in the population of athletes was approximately $47 \%$. The mechanisms responsible for post-exercise cTnT release and the kinetics of cTnT release after exercise are not clear, and whether postexercise cTnT release is related to microinjury of the myocardium remains to be determined.

\section{Inflammation}

A few studies have found that excessive training may lead to tissue injury, which activates circulating monocytes, producing large quantities of IL- $1 \beta$ and/or IL- 6 and/or tumour necrosis 

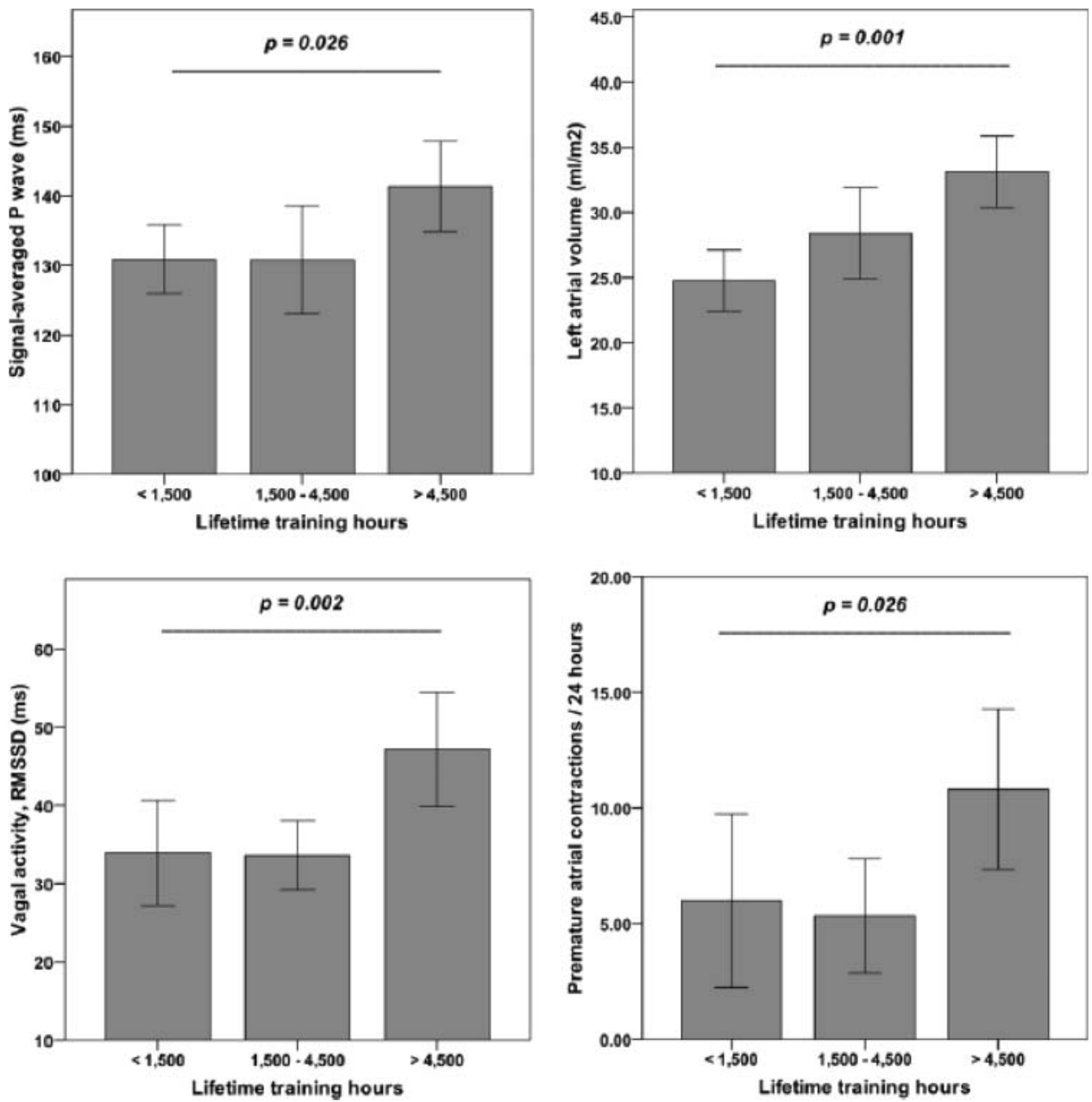

Figure 1 Signal-averaged P-wave duration, left atrial volume, vagal activity (expressed as root of mean squared differences of successive normal-to-normal intervals) and number of premature atrial contractions in $24 \mathrm{~h}$ stratified according to lifetime training hours ( $\mathrm{p}$ values for analysis of variance). Reproduced with permission from Winhelm et al. ${ }^{20}$

factor- $\alpha$ and systemic inflammation. ${ }^{42}$ Additionally, several studies have related an increase in C-reactive protein (CRP) and interleukins (ILs) in both paroxysmal and persistent $\mathrm{AF}^{44-46}$ Elevation of CRP and IL- 6 might also contribute to generation and perpetuation of $\mathrm{AF}$, as evidenced by marked inflammatory infiltrates, myocyte necrosis and fibrosis found in atrial biopsies of patients with $\mathrm{LAF}^{30}$ 43-45

Such observations suggest that sustained, intensive overtraining could produce a chronic inflammatory response in athletes that increases their risk for AF. However, there are no studies in the literature that confirm the association between AF, inflammation and exercise.

\section{Atrial remodelling}

Atrial remodelling has been shown to be present in elite athletes. Pelliccia et al ${ }^{21}$ reported that $20 \%$ of endurance sport athletes had larger left atrial dimensions compared to sedentary controls. Similarly, D'Andrea et al ${ }^{47}$ showed that LA enlargement is relatively common in top-level athletes. GIRAFA study data $^{16}$ showed that patients with LAF had a larger atrium than the controls. Furthermore, there were no differences in left atrial size between patients with a first episode of AF and those suffering recurrences, suggesting that structural changes were present before onset of AF. Grimsmo et $a l^{19}$ found that the left atrial diameter and the left atrial area were larger in the AF group and left atrial size was associated with LAF in endurancetrained athletes.

Similarly, an enlarged left atrium (atrial volume $>29.0 \mathrm{ml}$ / $\mathrm{m}^{2}$ ) was present in $24 \%$ of runners in the low-training group ( $<1500 \mathrm{~h}$ of lifetime training), $40 \%$ of runners in the mediumtraining group (1500-4500 h) and $83 \%$ of runners in the hightraining group ( $>4500 \mathrm{~h}$ of lifetime training) $(p=0.001) .{ }^{20}$

In addition, experimental studies of our group ${ }^{31}$ observed a left atrial dilatation, left ventricular hypertrophy and dilatation at 16 weeks of training, findings that are consistent with the features of the athlete's heart described in humans.

The reversibility of arrhythmogenic remodelling has also been assessed. A few studies have reported reversal of the remodelling associated with long-term exercise after long-term detraining. ${ }^{48}$ Our group evaluated whether a period of rest could allow reversion of the profibrotic changes induced by endurance training. ${ }^{31}$ The abnormal cardiac remodelling caused by 16 weeks of intensive exercise were reversed after 8 weeks of detraining.

The role of physical activity cessation and its effect upon AF will be further discussed in the text.

\section{CLINICAL CHARACTERISTICS OF SPORT-RELATED AF}

The usual clinical profile of sport-related AF is a middle-aged male athlete, with a history of long-term regular endurance 
sport practice who is currently involved in regular, highintensity endurance sport practice.

AF typically presents as a paroxysmal and highly symptomatic crisis, initially very occasional and self-limited, but becomes more frequent and prolonged over the years and can progress to persistent AF. Hoogsteen et al ${ }^{11}$ found that $17 \%$ exercise-related paroxysmal AF progressed to persistent AF and the GIRAFA study ${ }^{16}$ showed that $43 \%$ of patients with exercise-related AF were in persistent AF. Characteristically, AF episodes occur at night or after meals, revealing that AF may be related to increased vagal tone. ${ }^{16}$ The AF crisis frequently coexists with common AFI in many patients.

\section{MANAGEMENT OF AF IN ATHLETES}

A careful history of all the potential contributing factors should be taken. Medical conditions such as hyperthyroidism, pericarditis, Wolff-Parkinson-White syndrome, hypertrophic cardiomyopathy or long OT syndrome must be ruled out. Alcohol consumption and other substances such as caffeine, anabolic steroids, cocaine or sympathomimetics in cold medicines should be investigated and discontinued.

\section{Sport activity reduction}

Furlanello et $a^{48}$ described a good response to sport abstinence in top-level athletes with AF. Similarly, Hoogsteen et al ${ }^{11}$ showed that up to $30 \%$ of athletes experienced fewer episodes of AF by reducing sport activity. Therefore, the initial approach should be to recommend reducing physical activity. According to the Study Group on Sports Cardiology of the European Association for Cardiovascular Prevention and Rehabilitation, ${ }^{49}$ athletes in an early stage of paroxysmal AF should discontinue training for 2 months to stabilise sinus rhythm. The degree of improvement during this resting period will determine whether athletes are allowed to resume their training.

Task Force 7 of the 36 th Bethesda Conference ${ }^{50}$ recommends that athletes with asymptomatic AF in the absence of structural heart disease can be permitted to participate in any competitive sport, provided they maintain a ventricular rate that increases and slows appropriately and is comparable to that of a normal sinus response in relation to the level of activity, while receiving no therapy or therapy with AV nodal-blocking drugs. Asymptomatic athletes who have AF episodes lasting 5-15 s with no increase in duration during exercise can participate in all sports. Athletes should take medications that slow down the ventricular rate and should stop exercise training if there is a history of high ventricular rate or haemodynamic instability during AF. In these patients, we usually prescribe AV-node slowing agents despite moderate bradycardia during sinus rhythm.

\section{Pharmacological options}

Class I antiarrhythmic drugs can be initiated for the prevention of AF episodes once adequate ventricular rate control during exercise has been assured. However, although these drugs may prevent AF recurrences, AF can also be converted into AFl. ${ }^{51} 52$ Therefore, a combination with calcium channel blockers is recommended. ${ }^{5354}$

The 'pill-in-the pocket' approach with class I drugs is recommended in athletes with paroxysmal AF. Sport activity should be limited in these patients until at least one half-life of the antiarrhythmic drug has passed. ${ }^{49}$

\section{Pulmonary vein ablation}

Circumferential pulmonary vein ablation (CPVA) has been introduced in clinical practice as an effective and safe treatment of AF, especially in patients with paroxysmal AF and no structural heart disease. ${ }^{55}$ Recent data support the efficacy of ablation strategy in athletes. Furlanello et at ${ }^{56}$ described a $90 \%$ success after a mean of two ablation procedures in 20 athletes, with a significant improvement in all quality-of-life parameters and in particular in those pertinent to physical functioning.

We compared the effectiveness of CPVA between a population of endurance athletes (defined as those who performed regular endurance sport activity for at least $3 \mathrm{~h}$ per week for at least the 10 years immediately preceding the arrhythmia diagnosis) with LAF and other patients with $\mathrm{AF}^{57}$ There were no differences in CPVA effectiveness between the two groups (figure 2A). Furthermore, left atrial diameter and long-standing AF, but not endurance sport practice, were the only independent predictors of recurrence. More recently, Koopman et al ${ }^{58}$

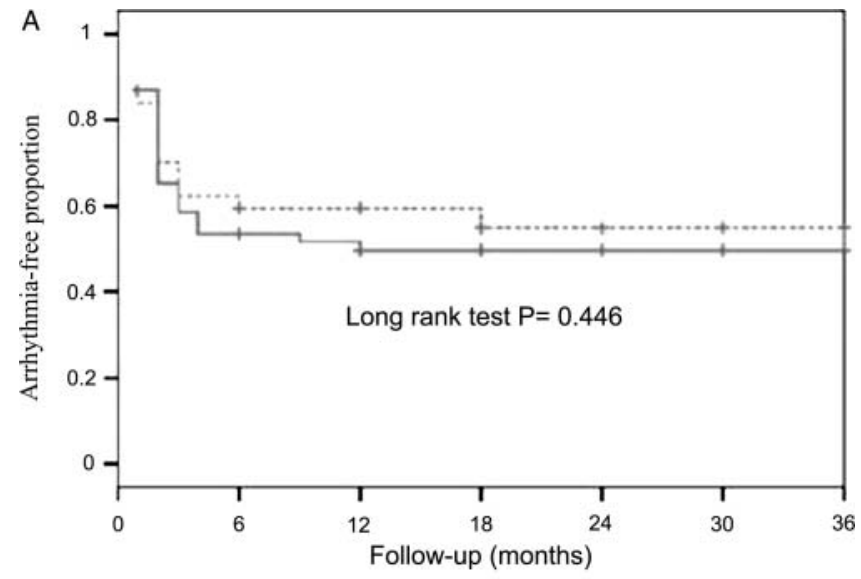

Number of patients at risk

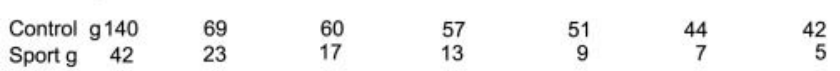

B

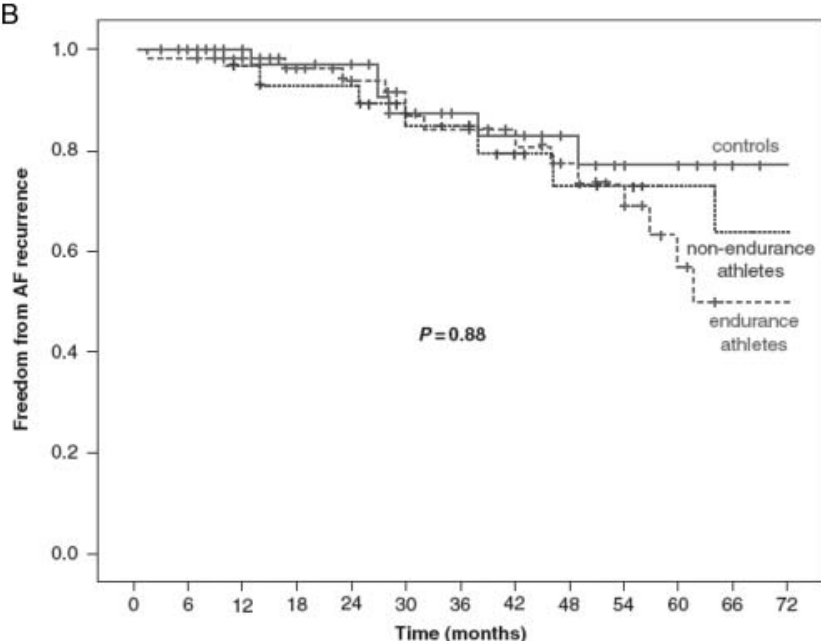

Number of patients at risk

$\begin{array}{llllllr}\text { controls } & 41 & 36 & 32 & 20 & 14 & 10 \\ \text { endurance } & 59 & 55 & 42 & 33 & 20 & 10 \\ \text { non-endurance } & 35 & 28 & 25 & 18 & 10 & 8\end{array}$

Figure 2 (A) Kaplan-Meier curves for long-term freedom from recurrent arrhythmias after a single ablation procedure in lone atrial fibrillation athletes group (dashed line) and control group (solid line). Reproduced with permission from Calvo et al. ${ }^{57}$ (B) Kaplan-Meier curves for final outcome after multiple ablations, on or off drugs. $A F$, atrial fibrillation. $p$ value: log-rank $p$ for 5 -year follow-up, endurance athletes versus non-endurance athletes versus controls. Reproduced with permission from Koopman et al. ${ }^{58}$ 
analysed the efficacy and success rate of radiofrequency catheter ablation for $\mathrm{AF}$ in an athlete population in comparison with contemporary controls. Athletes were defined as those who performed sport for $\geq 3 \mathrm{~h}$ per week during $\geq 10$ years or for a total of $\geq 1500 \mathrm{~h}$ after the age of 14 years. Compared with the control group, endurance and non-endurance athlete groups had a similar proportion of arrhythmia-free patients evaluated at 3 years after repeated ablation procedures (figure $2 \mathrm{~B}$ ).

After a successful ablation procedure and the absence of symptomatic recurrences for 3 months or more, resumption of all sports activity seems warranted, but the athletes should be followed up closely (ie, every 6 months). ${ }^{49}$

\section{Cavotricuspid isthmus ablation}

Ablation for AFl is a very effective intervention, with a high procedural success rate (90-98\%) and low recurrence rate (3-11\%). ${ }^{59-61}$ Non-competitive sports participation can be allowed early after ablation unless symptoms of major haemodynamic impairment were present during exercise before ablation, and the resumption of competitive sports activity is possible after a 3 -month period free of flutter. ${ }^{49}$

The success of the intervention is often offset by the development of AF. Heidbuchel et al ${ }^{14}$ reported that a history of endurance sports participation was significantly associated with an increased risk for AF development after flutter ablation. Therefore, in athletes suffering concomitant AF and AFl, the 'hybrid' therapy of class I drugs and the ablation of flutter or CPVI together with flutter ablation could be considered.

\section{Anticoagulation}

The need of anticoagulation is based on the presence of classic risk factors for thromboembolic events. ${ }^{62}$ Anticoagulation therapy excludes these individuals from sports with a risk of bodily collision or trauma. ${ }^{49}$

\section{CONCLUSIONS}

There is growing evidence that long-term endurance sports participation can result in cardiac structural changes and alterations in the autonomic system, which can result in the initiation and maintenance of $\mathrm{AF}$ and $\mathrm{AFl}$, although the mechanisms explaining the relationship between these conditions remain to be elucidated.

Reducing sports activities may need to be considered as part of the therapeutic advice to minimise the risk of AF or AFl development in endurance athletes. In addition, CPVA and AFI ablation have been shown to be as safe and effective as in general population and should be recommended in highly symptomatic and drug-refractory endurance athletes.

\section{Summary}

- Regular long-term endurance sports training may increase the risk for atrial flutter (AFI) and atrial fibrillation (AF).

- $\mathrm{AF}$ in athletes is initially paroxysmal, and most episodes have a vagal origin.

- Atrial ectopic beats, inflammatory changes, atrial enlargement with dilatation and fibrosis and increased vagal tone have been proposed as potential mechanisms for $\mathrm{AF}$ and $\mathrm{AFI}$ development in endurance athletes.

- The outcome of CPVA for AF in endurance athletes seems to be no different than in other patients.
Contributors Lluís Mont and Josep Brugada.

Patient consent Obtained.

Ethics approval Ethic Committee of Hospital Clínic of Barcelona.

Provenance and peer review Commissioned; externally peer reviewed.

\section{REFERENCES}

1. Go AS, Hylek EM, Phillips KA, et al. Prevalence of diagnosed atrial fibrillation in adults: national implications for rhythm management and stroke prevention: the AnTicoagulation and Risk Factors in Atrial Fibrillation (ATRIA) Study. JAMA 2001;285:2370-5.

2. Furberg CD, Psaty BM, Manolio TA, et al. Prevalence of atrial fibrillation in elderly subjects (the Cardiovascular Health Study). Am J Cardiol 1994;74:236-41.

3. Benjamin EJ, Wolf PA, D'Agostino RB, et al. Impact of atrial fibrillation on the risk of death: the Framingham Heart Study. Circulation 1998;98:946-52.

4. Fuster V, Rydén LE, Cannom DS, et al. ACC/AHA/ESC 2006 Guidelines for the Management of Patients with Atrial Fibrillation-Executive Summary. A Report of the American College of Cardiology/American Heart Association Task Force on Practice Guidelines and the European Society of Cardiology Committee for Practice Guidelines (Writing Committee to Revise the 2001 Guidelines for the Management of Patients With Atrial Fibrillation). J Am Coll Cardiol 2006;48:854-906.

5. Planas F, Romero-Menor C, Gabriel Vázquez-Oliva JS, et al, on behalf of the FAP Study researchers. Perfil clínico de la fibrilación auricular paroxística primaria (registro FAP). Rev Esp Cardiol 2001;54:838-44.

6. Lévy S, Maarek M, Coumel $P$, et al. Characterization of different subsets of atrial fibrillation in general practice in France: the ALFA study. The College of French Cardiologists. Circulation 1999;99:3028-35.

7. Morris JN, Everitt MG, Pollard R, et al. Vigorous exercise in leisure-time: protection against coronary heart disease. Lancet 1980;2:1207-10.

8. Kujala UM, Kaprio J, Taimela S, et al. Prevalence of diabetes, hypertension, and ischemic heart disease in former elite athletes. Metabolism 1994;43:1255-60.

9. Karjalainen J, Kujala UM, Kaprio J, et al. Lone atrial fibrillation in vigorously exercising middle aged men: case-control study. BMJ 1998;316:1784-5

10. Mont L, Sambola A, Brugada J, et al. Long lasting sport practice and atria fibrillation. Eur Heart J 2002;23:477-82.

11. Hoogsteen J, Schep G, van Hemel NM, et al. Paroxysmal atrial fibrillation in male endurance athletes. A 9-year follow up. Europace 2004;6:222-8.

12. Elosua $\mathbf{R}$, Arquer $A$, Mont $L$, et al. Sport practice and the risk of lone atrial fibrillation: a case-control study. Int J Cardiol 2005;108:332-7.

13. Baldesberger S, Bauersfeld U, Candinas R, et al. Sinus node disease and arrhythmias in the long term follow-up of former professional cyclists. Eur Heart $J$ 2008:29:71-8.

14. Heidbuchel $\mathbf{H}$, Anne W, Willems R, et al. Endurance sport is a risk factor for atrial fibrillation after ablation for atrial flutter. Int J Cardiol 2006;107:67-72.

15. Molina L, Mont L, Marrugat J, et al. Long-term endurance sport practice increases the incidence of lone atrial fibrillation in men: a follow-up study. Europace 2008;10:618-23.

16. Mont L, Tamborero D, Elosua R, et al. on behalf of the GIRAFA (Grup Integrat de Recerca en Fibril-lacio Auricular) Investigators. Physical activity, height and left atrial size are independent risk factors for lone atrial fibrillation in middle-aged healthy individuals. Europace 2008;10:15-20.

17. Abdulla J, Nielsen JR. Is the risk of atrial fibrillation higher in athletes than in the general population? A systematic review and meta-analysis. Europace 2009;11:1156-9.

18. Aizer A, Gaziano JM, Cook NR, et al. Relation of vigorous exercise to risk of atrial fibrillation. Am J Cardiol 2009;103:1572-7e7.

19. Grimsmo J, Grundvold I, Maehlum S, et al. High prevalence of atrial fibrillation in long-term endurance cross-country skiers: echocardiographic findings and possible predictors - a 28-30 years follow- up study. Eur J Cardiovasc Prev Rehabil 2010:17:100-5.

20. Winhelm $\mathbf{M}$, Roten $\mathrm{L}$, Tanner $\mathrm{H}$, et al. Atrial remodeling, autonomic tone, and lifetime training hours in nonelite athletes. Am J Cardiol 2011;108:580-5.

21. Pelliccia A, Maron BJ, Di Paolo FM, et al. Prevalence and clinical significance of left atrial remodeling in competitive athletes. J Am Coll Cardiol 2005;46:690-6.

22. Mozaffarian D, Furberg CD, Psaty BM, et al. Physical activity and incidence of atrial fibrillation in older adults. The Cardiovascular Health Study. Circulation 2008;118:800-7.

23. Haissaguerre $\mathbf{M}$, Jais $\mathrm{P}$, Shah $\mathrm{DC}$, et al. Spontaneous initiation of atrial fibrillation by ectopic beats originating in the pulmonary veins. $N$ Engl J Med 1998;339:659-66.

24. Bjornstad H, Storstein L, Meen HD, et al. Ambulatory electrocardiographic findings in top athletes, athletic students and control subjects. Cardiology 1994;84:42-50.

25. Bettomi M, Zimmermann M. Autonomic tone variations before the onset of paroxysmal atrial fibrillation. Circulation 2002;105:2753-9.

26. Amar D, Zhang H, Miodownik S, et al. Competing autonomic mechanisms precede the onset of postoperative atrial fibrillation. JACC 2003:42:1262-70. 
27. Ogawa M, Zhou S, Tan AY, et al. Left stellate ganglion and vagal nerve activity and cardiac arrhythmias in ambulatory dogs with pacing-induced congestive heart failure. J Am Coll Cardiol 2007;50:335-43.

28. Patterson E, Po SS, Scherlag BJ, et al. Triggered firing in pulmonary veins initiated by in vitro autonomic nerve stimulation. Heart Rhythm 2005;2:632-3.

29. Coumel P. Paroxysmal atrial fibrillation: a disorder of autonomic tone? Eur Heart $J$ 1994;15:9-16.

30. Frustaci A, Chimenti C, Bellocci $F$, et al. Histological substrate of atrial biopsies in patients with lone atrial fibrillation. Circulation 1997;96:1180-4.

31. Benito B, Gay-Jordi G, Serrano A, et al. Cardiac arrhythmogenic remodeling in a rat model of long-term intensive exercise training. Circulation 2011;123:13-22.

32. Lindsay MM, Dunn FG. Biochemical evidence of myocardial fibrosis in veteran endurance athletes. Br J Sports Med 2007;41:447e52.

33. Breuckmann F, Möhlenkamp S, Nassenstein K, et al. Myocardial late gadolinium enhancement: prevalence, pattern, and prognostic relevance in Marathon runners. Radiology 2009;251:50-7.

34. Wilson M, O'Hanlon R, Prasad S, et al. Diverse patterns of myocardial fibrosis in lifelong, veteran endurance athletes. J Appl Physiol 2011;110:1622-6.

35. Neumayr G, Pfister R, Mitterbauer G, et al. Effect of the "Race Across The Alps" in elite cyclists on plasma cardiac troponins I and T. Am J Cardiol 2002;89:484-6.

36. Rifai N, Douglas PS, O'Toole M, et al. Cardiac troponin T and I, echocardiographic [correction of electrocardiographic] wall motion analyses, and ejection fractions in athletes participating in the Hawaii Ironman Triathlon. Am J Cardiol 1999;83:1085-9.

37. Scharhag J, Herrmann M, Urhausen A, et al. Independent elevations of Nterminal pro-brain natriuretic peptide and cardiac troponins in endurance athletes after prolonged strenuous exercise. Am Heart J 2005;150:1128-34.

38. Shave R, Whyte G, George D, et al. Prolonged exercise should be considered alongside typical symptoms of acute myocardial infarction when evaluating increases in cardiac troponin T. Heart 2005;91:1219-20.

39. Shave R, Dawson E, Whyte $G$, et al. Cardiac troponin $T$ in female athletes during a two-day mountain marathon. Scott Med J 2003;48:41-2.

40. Shave R, Dawson E, Whyte G, et al. Evidence of exercise induced cardiac dysfunction and elevated cTnT in separate cohorts competing in an ultra-endurance mountain marathon race. Int J Sports Med 2002;23:489-94.

41. Shave R, George K, Atkinson G, et al. Exercise-induced cardiac troponin T release: a meta-analysis. Med Sci Sports Exerc 2007;39:2099-106.

42. Smith LL. Cytokine hypothesis of overtraining: a physiological adaptation to excessive stress? Med Sci Sports Exerc 2000;32:317-31.

43. Korantzopoulos P, Kolettis T, Siogas K, et al. Atrial fibrillation and electrical remodeling: the potential role of inflammation and oxidative stress. Med Sci Monit 2003;9:RA225-9.

44. Aviles R, Martin D, Apperson-Hansen C, et al. Inflammation as a risk factor for atrial fibrillation. Circulation 2003:108:3006-10.

45. Chung MK, Martin DO, Sprecher D, et al. C-reactive protein elevation in patients with atrial arrhythmias. Circulation 2001;104:2886-91.

46. Psychari S, Apostolou T. Relation of elevated C-reactive protein and interleukin-6 levels to left atrial size and duration of episodes in patients with atrial fibrillation. Am J Cardiol 2005;95:764-7.
47. D'Andrea A, Riegler L, Cocchia $\mathrm{R}$, et al. Left atrial volume index in highly trained athletes. Am Heart J 2010;159:1155-61.

48. Furlanello F, Bertoldi A, Dallago $\mathrm{M}$, et al. Atrial fibrillation in elite athletes. $J$ Cardiovasc Electrophysiol 1998;9:S63-8.

49. Heidbuchel H, Panhuyzen-Goedkoop N, Corrado D, et al. Recommendations for participation in leisure-time physical activity and competitive sports in patients with arrhythmias and potentially arrhythmogenic conditions Part I: Supraventricular arrhythmias and pacemakers. Eur J Cardiovasc Prev Rehabil 2006;13 475-84.

50. Zipes D, Ackerman MJ, Estes M, et al. Eligibility recommendations for competitive athletes with cardiovascular abnormalities-General considerations. Task Force 7: arrhythmia. J Am Coll Cardiol 2005;45:1354-63.

51. Kawabata M, Hirao K, Horikawa T, et al. Syncope in patients with atrial flutter during treatment with class Ic antiarrhythmic drugs. J Electrocardiol 2001;34:65-72.

52. Brembilla-Perrot B, Houriez $P$, Beurrier $D$, et al. Predictors of atrial flutter with $1: 1$ conduction in patients treated with class I antiarrhythmic drugs for atrial tachyarrhythmias. Int J Cardiol 2001;80:7-15

53. Nabar A, Rodriguez LM, Timmermans $C$, et al. Effect of right atrial isthmus ablation on the occurrence of atrial fibrillation: observations in four patient groups having type I atrial flutter with or without associated atrial fibrillation. Circulation 1999;99:1441-5.

54. Reithmann C, Dorwarth U, Dugas M, et al. Risk factors for recurrence of atrial fibrillation in patients undergoing hybrid therapy for antiarrhythmic drug-induced atria flutter. Eur Heart J 2003;24:1264-72.

55. Cappato R, Calkins H, Chen SA, et al. Updated worldwide survey on the methods, efficacy, and safety of catheter ablation for human atrial fibrillation. Circ Arrhythm Electrophysiol 2010;3:32-8.

56. Furlanello $\mathbf{F}$, Lupo $\mathrm{P}$, Pittalis $\mathrm{M}$, et al. Radiofrequency catheter ablation of atrial fibrillation in athletes referred for disabling symptoms preventing usual training schedule and sport competition. J Cardiovasc Electrophysiol 2008;19: 457-62.

57. Calvo N, Mont L, Tamborero D, et al. Efficacy of circumferential pulmonary vein ablation of atrial fibrillation in endurance athletes. Europace 2010;12:30-6.

58. Koopman P, Nuyens D, Garweg C, et al. Efficacy of radiofrequency catheter ablation in athletes with atrial fibrillation. Europace 2011;13:1386-93.

59. Hsieh MH, Tai CT, Chiang CE, et al. Recurrent atrial flutter and atrial fibrillation after catheter ablation of the cavotricuspid isthmus: a very long-term follow-up of 333 patients. J Interv Card Electrophysiol 2002;7:225-31.

60. Reithmann C, Dorwarth U, Dugas M, et al. Risk factors for recurrence of atrial fibrillation in patients undergoing hybrid therapy for antiarrhythmic drug-induced atria flutter. Eur Heart J 2003:24:1264-72.

61. Schmieder S, Ndrepepa G, Dong J, et al. Acute and long-term results of radiofrequency ablation of common atrial flutter and the influence of the right atria isthmus ablation on the occurrence of atrial fibrillation. Eur Heart J 2003;24: 956-62.

62. Reynolds MW, Fahrbach K, Hauch 0, et al. Warfarin anticoagulation and outcomes in patients with atrial fibrillation: a systematic review and metaanalysis. Chest 2004:126:1938-45. 